\title{
Editorial for special issue on serious games and education
}

\author{
Fotis Liarokapis ${ }^{1} \cdot$ Kurt Debattista $^{2}$
}

Published online: 27 October 2017

(C) Springer Science+Business Media New York 2017

Serious games are non-leisure applications of gaming technologies and allow for educational and social interactions. The applications of these technologies are very broad and this special issue has attracted contributions from areas such as gamification, narratives, game design, simulation and pedagogy to give a few examples. The ambition of this special issue is to trigger debate and discussion around these key issues and themes and to continue to frame future research.

In particular, this special issue is focused on serious games and education and is based on the best papers received for the 7th International Conference on Virtual Worlds and Games for Serious Applications (VS-GAMES 2015) which took place in Skövde, Sweden between the 16th to 18th of September, 2015. The conference had 48 submissions from which nine papers were originally invited to the journal. After two rounds of reviews, the four strongest papers were selected for this special issue.

These papers selected are entitled: "Gamification: a systematic review of design frameworks", "Linking Serious Game Narratives with Pedagogical Theories and Pedagogical Design Strategies", "Inclusive Game Design Facilitating Shared Gaming Experience" and "Project Sanitarium: Playing Tuberculosis to Its End Game".

Mora et al. (2017) presented a systematic review of the gamification design frameworks providing a useful resource to educational practitioners as well as gamification designers and researchers. A total of 2314 unique works were initially recorded, and after applying a systematic filtering process, a definitive list of 40 works was analysed. The paper also presents an assessment of the main features found in the discussed approaches.

De Troyer et al. (2017) illustrated how the Domain Specific Modeling Language ATTAC-L, for modeling the narrative of a serious game, allows for creating a link

Fotis Liarokapis

K.Debattista@warwick.ac.uk

$1 \quad$ Masaryk University, Brno, Czech Republic

2 University of Warwick, Coventry, UK 
between the processes of pedagogical design and narrative modeling by means of an elaborate annotation system. They show how the annotation system can be used to document and integrate the use of a well-grounded pedagogical theory, Social Cognitive Theory (SCT), for achieving the goals of a serious game, as well as when the serious game is part of a program developed by means of a well-grounded design strategy, Intervention Mapping Protocol (IMP).

Wilhelmsson et al. (2017) presented the results from a study comparing the perception and understanding of a game story between sighted and visually impaired players playing the same game. In particular, whether sighted and visually impaired players could experience and recount the same story construed from the plot elements that are either manifested by audio and graphics in the case of sighted players or primarily by audio in the case of visually impaired players. The study shows that the perception of the story was almost identical between the two groups.

Donald et al. (2017) presented Project Sanitarium which aimed to educate students through a workplace simulation pedagogical model, encourage public engagement at events and through news coverage, and, lastly, to prototype whether games could be used to simulate a virtual clinical trial. The serious game incorporates a mathematical model that is based on data from real-world drug trials. The interdisciplinary pedagogical model provides undergraduates with workplace simulation, wider industry collaboration and access to academic expertise to solve challenging and complex problems.

\section{References}

De Troyer, O., Van Broeckhoven, F., \& Vlieghe, J. (2017). Linking serious game narratives with pedagogical theories and pedagogical design strategies. Journal of Computing in Higher Education. doi:10.1007/s12528-017-9142-4.

Donald, I., Meyer. K. A., Brengman, J., Gillespie, S. H., \& Bowness, R. (2017). Project sanitarium: Playing tuberculosis to its end game. Journal of Computing in Higher Education. doi:10.1007/ s12528-017-9145-1.

Mora, A., Riera, D., González, C., \& Arnedo-Moreno, J. (2017). Gamification: A systematic review of design frameworks. Journal of Computing in Higher Education. doi:10.1007/s12528-017-9150-4.

Wilhelmsson, U., Engström, H., Brusk, J., \& Östblad, P. A. (2017). Inclusive game design facilitating shared gaming experience. Journal of Computing in Higher Education. doi:10.1007/s12528-0179146-0.

Fotis Liarokapis is an Associate Professor at the HCI Laboratory at Masaryk University, Brno, Czech Republic. He has a PhD in Computer Engineering from the University of Sussex, an MSc in Computer Science from the University of Hull and a BEng in Computer Systems Engineering from the University of Sussex. He has organised multiple conferences and workshops and he is the co-founder of the IEEE VSGames international conference.

Kurt Debattista is an Associate Professor at the University of Warwick, Coventry, UK. He has a PhD from the University of Bristol, an MSc in Psychology, an MSc in Computer Science and a BSc in Mathematics and Computer Science. His research interests include HDR imaging, high-fidelity computer graphics, serious games and parallel computing. 